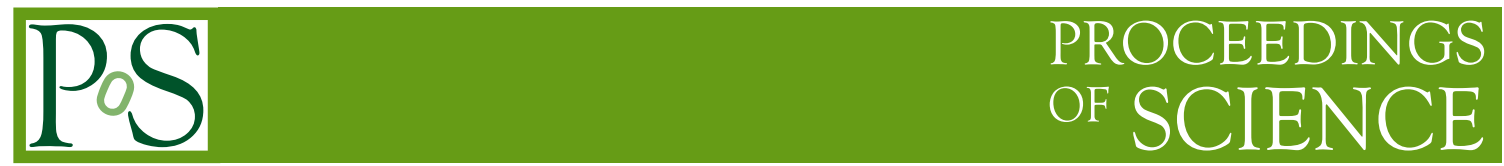

\title{
CP violation in Charm with the LHCb experiment
}

\author{
Serena Maccolini* \\ University of Bologna and INFN, \\ Bologna, Italy \\ E-mail: serena.maccolini@cern.ch
}

Measurements of charge-parity $(C P)$ violation and mixing in charm provide a precise test of the Standard Model and can potentially probe interactions beyond the Standard Model. The $\mathrm{LHCb}$ experiment has provided the most precise measurements to date in this scope. We present recent results obtained during the current year, including the first observation of a non-zero mass difference between the neutral charm eigenstates - a new milestone in charm physics.

The Ninth Annual Conference on Large Hadron Collider Physics - LHCP2021

7-12 June 2021

Online

${ }^{*}$ Speaker 


\section{Introduction}

In the Standard Model (SM) of particle physics, $C P$ violation (CPV) is introduced through an irreducible complex phase in the Cabibbo-Kobayashi-Maskawa (CKM) mixing matrix [1]. The smallness of the elements of the CKM matrix involved suppresses the expectations for CPV in charm at a level typically below $10^{-3}$ [2] and makes $C P$ violation in charm sensitive to possible contributions of physics beyond the SM. Most importantly, the charm-quark sector offers a unique opportunity to test the CKM formalism, since it provides access to operators that affect only uptype quarks, while leaving the strange and beauty hadrons unaffected. In March 2019, the LHCb collaboration announced the first observation of CPV in the decay of charm hadrons [3]. However, the interpretation of this observation is unclear, since theoretical predictions are difficult to compute reliably due to non-perturbative quantum-chromodynamics effects [4-6]. Further studies of direct $\mathrm{CPV}$ as well as mixing and time-dependent CPV in charm decays are needed to clarify the picture.

\section{Observation of the mass difference between neutral charm-meson eigenstates with $D^{0} \rightarrow K_{\mathrm{S}}^{0} \pi^{-} \pi^{+}$decays}

The splitting of the masses $\left(m_{1,2}\right)$ and decay widths $\left(\Gamma_{1,2}\right)$ of the neutral charm-meson eigenstates, where $\left|D_{1,2}\right\rangle \equiv p\left|D^{0}\right\rangle \pm q\left|\bar{D}^{0}\right\rangle$ with $|p|^{2}+|q|^{2}=1$, governs the oscillations of $D^{0}$ mesons and can be conveniently parametrized through the mixing parameters $x \equiv\left(m_{1}-m_{2}\right) /(\Gamma)$ and $y \equiv\left(\Gamma_{1}-\Gamma_{2}\right) /(2 \Gamma)$, where $\Gamma=\left(\Gamma_{1}+\Gamma_{2}\right) / 2$. The rich resonance structure of $D^{0} \rightarrow K_{\mathrm{S}}^{0} \pi^{-} \pi^{+}$decays implies the presence of large strong phases that vary across the Dalitz plane and, consequently, provides good sensitivity to all mixing and CP violation parameters. However, the decay dynamics of this three-body decay and the variations of the detector efficiency across the Dalitz plane as a function of decay time need to be modeled carefully in order to take advantage of this feature. Both these challenges are mitigated by a model-independent analysis procedure proposed in Ref. [7], the so-called "Bin-Flip method". This consists in dividing the Dalitz plane into two sets of regions, symmetrically distributed with respect to the bisector $m_{+}^{2}=m_{-}^{2}$, where $m_{ \pm}^{2}$ is equal to $m^{2}\left(K_{\mathrm{S}}^{0} \pi^{ \pm}\right)$for $D^{0}$ decays and to $m^{2}\left(K_{\mathrm{S}}^{0} \pi^{\mp}\right)$ for $\bar{D}^{0}$ decays, chosen so as to keep the strong-phase difference $(\Delta \delta)$ between $D^{0}$ and $\bar{D}^{0}$ decays approximately constant within each region. The binning, together with Dalitz plot distribution, is displayed in Fig. 1. The lower part of the Dalitz plane is dominated by unmixed, Cabibbo-favored $D^{0}$ decays, while in the upper part of the Dalitz plane the contribution of Cabibbo-favored decays following mixing becomes more and more important with respect to the unmixed doubly Cabibbo-suppressed decays as decay time increases. For each $D^{0}$ decay time, and each spatial region of constant strong-phase difference, labelled $\pm b$, the ratio of the number of decays in the in the upper Dalitz-plot bin $(-b)$ to its lower counterpart $(+b)$ is approximately equal to

$$
R_{b}(t)^{ \pm} \approx r_{b}-\sqrt{r_{b}}\left[\left(1-r_{b}\right) c_{b} y-\left(1+r_{b}\right) s_{b} x\right] t / \tau_{D^{0}},
$$

where $\tau_{D^{0}}$ is the lifetime of the $D^{0}$ meson, $R_{b}^{ \pm}$corresponds to the yield ratio for initially produced $D^{0}\left(\bar{D}^{0}\right)$ mesons, $r_{b}$ is the value of $R_{b}$ at zero decay time, and $c_{b}$ and $s_{b}$ are the average cosine and sine of the strong-phase $d$ difference between positive and negative Dalitz-plot bins and are based on external inputs [8]. A previous LHCb measurement [9] using the full LHCb Run 1 dataset 

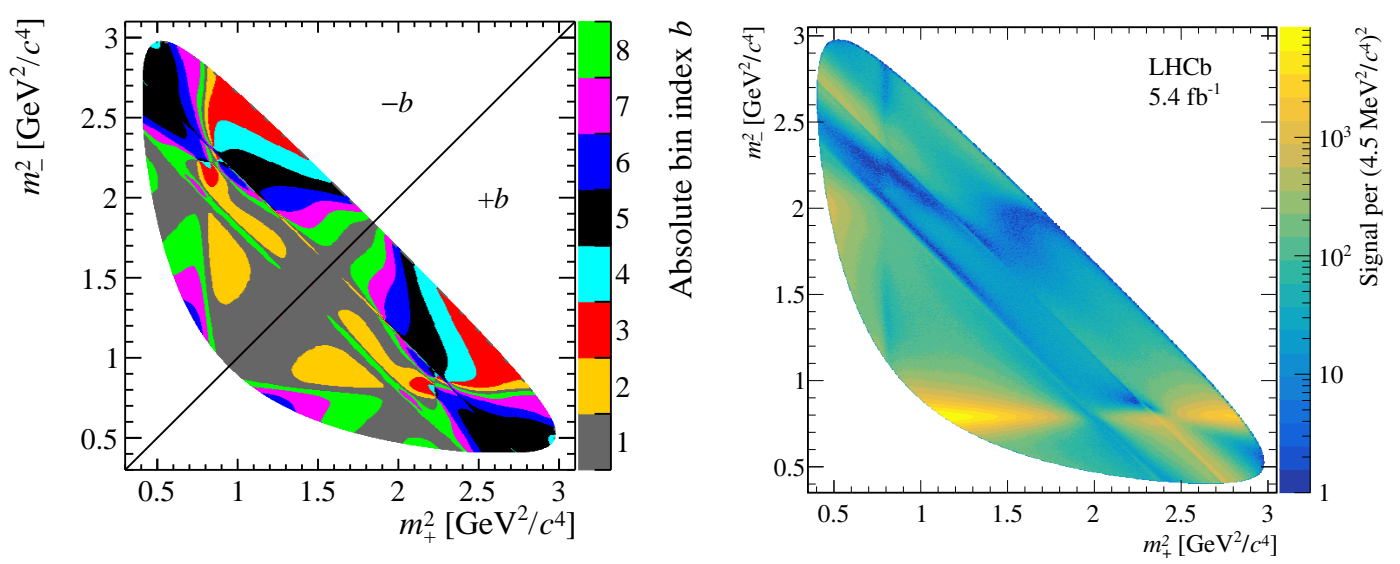

Figure 1: Left: Iso- $\Delta \delta$ binning of the $D^{0} \rightarrow K_{\mathrm{S}}^{0} \pi^{-} \pi^{+}$Dalitz plot, based on the BaBar 2008 amplitude model [8]. Positive indices refer to bins in the lower region, $m_{+}^{2}>m_{-}^{2}$; negative indices refer to those in the upper region, $m_{+}^{2}<m_{-}^{2}$. Colors indicate the absolute value of the bin index $b$. Right: Dalitz plot of the $D^{0} \rightarrow K_{\mathrm{S}}^{0} \pi^{-} \pi^{+}$decay.

(2011-2012, corresponding to an integrated luminosity of $3 \mathrm{fb}^{-1}$ ) gave the first evidence of a nonzero mass difference between neutral charm-meson eigenstates, reporting $x=\left(3.9_{-1.2}^{+1.1}\right) \cdot 10^{-3}$. The analysis presented in this document relies on the LHCb Run 2 dataset (2016-2018, corresponding to an integrated luminosity of $5.4 \mathrm{fb}^{-1}$ ) [10]. $D^{0}$ candidates are obtained from prompt $D^{*+} \rightarrow D^{0} \pi^{+}$ decays, where the charge of the pion tags the flavour of the $D^{0}$. The fits to the time-dependent yield ratios (Eq. 1) for each region $b$ are shown in Fig. 2. For the $C P$-averaraged yield rations (left plots), deviations from constant values are due to mixing. The right plots display the differences of ratios between $D^{0}$ and $\bar{D}^{0}$ decays, where a significant slope, would be due to $C P$ violation. The results

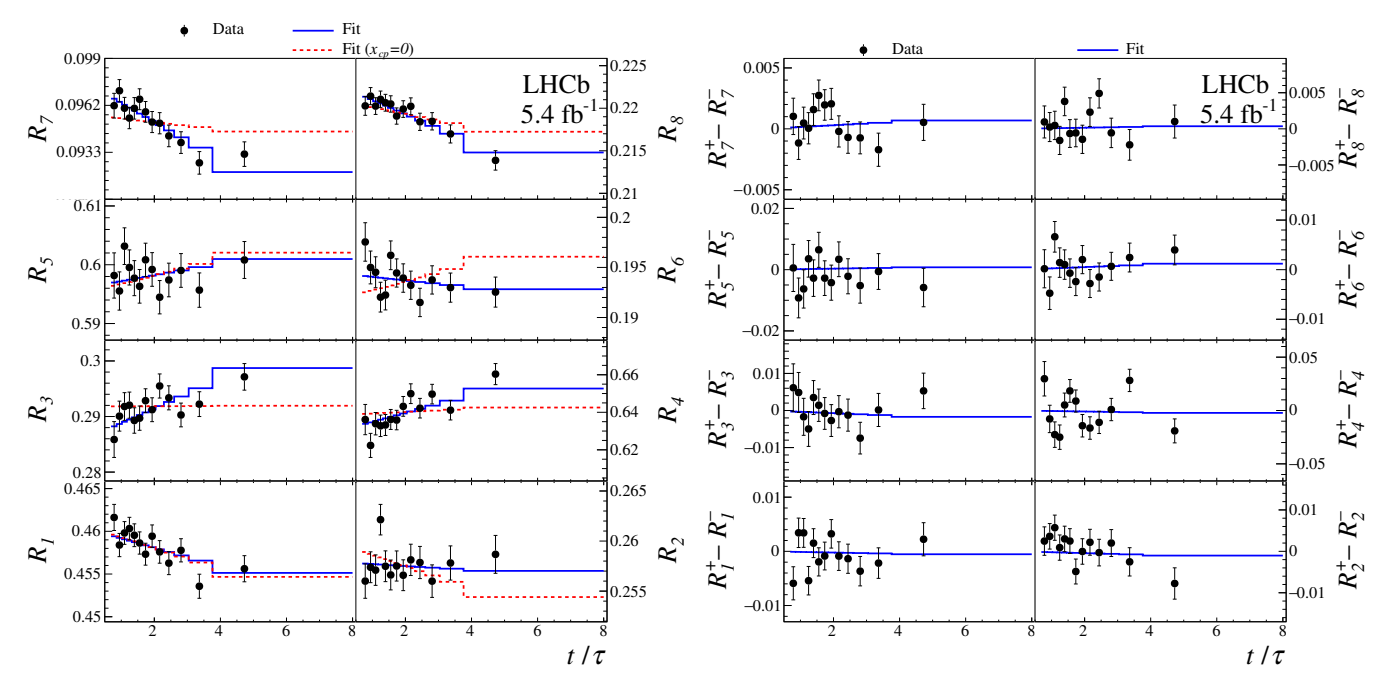

Figure 2: Left: Time-dependent $C P$-averaged yield ratios of initially-produced $D^{0}$ and $\bar{D}^{0}$ candidates for each of the 8 Dalitz-plots bins. Right: Differences of $D^{0}$ and $\bar{D}^{0}$ yield ratios. Fit projections are overlaid. 
for the mixing and $C P$ violation parameters $x, y,|q / p|$ and $\phi \sim \arg (q / p)$ are

$$
\begin{aligned}
x & =\left(3.98_{-0.54}^{+0.56}\right) \cdot 10^{-3}, \\
y & =\left(4.6_{-1.4}^{+1.5}\right) \cdot 10^{-3}, \\
|q / p| & =0.996 \pm 0.052, \\
\phi & =\left(-0.056_{-0.051}^{+0.047}\right) \mathrm{rad} .
\end{aligned}
$$

The parameter $x$ is found to be inconsistent with zero with a statistical significance of seven standard deviations. This consitutes the world's first observation of a non-zero mass difference between neutral charm-meson eigenstates.

\section{Other searches for $C P$ violation in charm decays}

Cabibbo-suppressed $D \rightarrow f$ decays provide the most sensitive tests of $C P$ violation, through the measurement of the time-dependent and time-integrated $C P$ asymmetry between the $D$ and $\bar{D}$ meson decay rates,

$$
A_{C P}(f, t)=\frac{\Gamma(D \rightarrow f, t)-\Gamma(\bar{D} \rightarrow \bar{f}, t)}{\Gamma(D \rightarrow f, t)+\Gamma(\bar{D} \rightarrow \bar{f}, t)} .
$$

In the case of $D^{0} \rightarrow f$, where the final state $f=K^{-} K^{+}$or $\pi^{-} \pi^{+}$is common to $D^{0}$ and $\bar{D}^{0}$ mesons, the asymmetry can be expandend to linear order in the mixing parameters as

$$
A_{C P}(D \rightarrow f, t) \approx a_{f}^{d}+\Delta Y_{f} t / \tau_{D^{0}},
$$

where $a_{f}^{d}$ is the $C P$ asymmetry in the decay and $\Delta Y_{f}$ quantifies time-depentent $C P$-violating effects. In the case of charged charm mesons, $A_{C P}(D \rightarrow f)$ is constant as a function of the decay time. The latest experimental measurements in this field, performed using the full LHCb Run 2 dataset (2015-2018, corresponding to an integrated luminosity of $5.7 \mathrm{fb}^{-1}$ ) [11-13], are

$$
\begin{aligned}
\Delta Y_{K K} & =(-2.3 \pm 1.5 \pm 0.3) \cdot 10^{-4}, \\
\Delta Y_{\pi \pi} & =(-4.0 \pm 2.8 \pm 0.4) \cdot 10^{-4} \\
A_{C P}\left(D^{+} \rightarrow \pi^{+} \pi^{0}\right) & =(-1.3 \pm 0.9 \pm 0.6) \% \\
A_{C P}\left(D^{+} \rightarrow K^{+} \pi^{0}\right) & =(-3.2 \pm 4.7 \pm 2.1) \% \\
A_{C P}\left(D^{+} \rightarrow \pi^{+} \eta\right) & =(-0.2 \pm 0.8 \pm 0.4) \% \\
A_{C P}\left(D^{+} \rightarrow K^{+} \eta\right) & =(-6 \pm 10 \pm 4) \% \\
A_{C P}\left(D_{s}^{+} \rightarrow K^{+} \pi^{0}\right) & =(-0.8 \pm 3.9 \pm 1.2) \% \\
A_{C P}\left(D_{s}^{+} \rightarrow \pi^{+} \eta\right) & =(0.8 \pm 0.7 \pm 0.5) \% \\
A_{C P}\left(D_{s}^{+} \rightarrow K^{+} \eta\right) & =(0.9 \pm 3.7 \pm 1.1) \% \\
A_{C P}\left(D^{0} \rightarrow K_{\mathrm{s}}^{0} K_{\mathrm{s}}^{0}\right) & =(-3.1 \pm 1.2 \pm 0.4) \%
\end{aligned}
$$

where the first uncertainties are statistical and the second are systematic. All results are found to be compatible with the conservation of $C P$ symmetry.

These new measurements constitute the world's most precise determinations of those quantities with the exception of the $C P$ asymmetries $A_{C P}\left(D_{s}^{+} \rightarrow \pi^{+} \eta\right)$ and $A_{C P}\left(D_{s}^{+} \rightarrow K^{+} \eta\right)$. In the latters, however, an outstanding result is achieved if the hadronic nature of the collider is taken into account. 


\section{References}

[1] M. Kobayashi and T. Maskawa, CP violation in the renormalizable theory of weak interaction, Prog. Theor. Phys. 49 (1973) 652.

[2] Y. Grossman, A. L. Kagan and Y. Nir, New physics and CP violation in singly Cabibbo suppressed D decays, Phys. Rev. D75 (2007) 036008 [hep-ph/0609178].

[3] LHCb collaboration, Observation of CP violation in charm decays, Phys. Rev. Lett. 122 (2019) 211803 LHCb-PAPER-2019-006 CERN-EP-2019-042, [1903 . 08726].

[4] M. Chala, A. Lenz, A. V. Rusov and J. Scholtz, $\Delta A_{C P}$ within the Standard Model and beyond, JHEP 07 (2019) 161 [1903. 10490].

[5] H.-N. Li, C.-D. Lü and F.-S. Yu, Implications on the first observation of charm CPV at $L H C b, 1903.10638$.

[6] A. Soni, Resonance enhancement of charm CP, 1905.00907.

[7] A. Di Canto, J. Garra Ticó, T. Gershon, N. Jurik, M. Martinelli, T. Pilař et al., Novel method for measuring charm-mixing parameters using multibody decays, Phys. Rev. D99 (2019) 012007 [1811.01032].

[8] CLEO collaboration, Model-independent determination of the strong-phase difference between $D^{0}$ and $\bar{D}^{0} \rightarrow K_{S, L}^{0} h^{+} h^{-}(h=\pi, K)$ and its impact on the measurement of the CKM angle $\gamma / \phi_{3}$, Phys. Rev. D82 (2010) 112006 [1010 . 2817].

[9] LHC $b$ collaboration, Measurement of the mass difference between neutral charm-meson eigenstates, Phys. Rev. Lett. 122 (2019) 231802 LHCb-PAPER-2019-001

CERN-EP-2019-032, [1903.03074].

[10] LHCb collaboration, Observation of the mass difference between neutral charm-meson eigenstates, Phys. Rev. Lett. 127 (2021) 111801 LHCb-PAPER-2021-009,

CERN-EP-2021-099, [2106.03744].

[11] LHC $b$ collaboration, Search for time-dependent $C P$ violation in $D^{0} \rightarrow K^{+} K^{-}$and $D^{0} \rightarrow \pi^{+} \pi^{-}$decays, 2105 .09889 LHCb-PAPER-2020-045,CERN-EP-2021-060, [2105.09889].

[12] LHC $b$ collaboration, Search for CP violation in $D_{(s)}^{+} \rightarrow h^{+} \pi^{0}$ and $D_{(s)}^{+} \rightarrow h^{+} \eta$ decays, JHEP 06 (2021) 019 LHCb-PAPER-2021-001,CERN-EP-2021-036, [2103.11058].

[13] LHCb collaboration, Measurement of CP asymmetry in $D^{0} \rightarrow K_{S}^{0} K_{S}^{0}$ decays, Phys. Rev. D104 (2021) 031102 LHCb-PAPER-2020-047,CERN-EP-2021-058, [2105 . 01565]. 